\title{
Vida familiar y música en el espacio social urbano. Programas narrativos e Ingeniería en Comunicación Social
}

Family life and music in the urban social space. Narrative programs and Engineering in Social Communication

Gerardo Guillermo León Barrios gleon@uabc.edu.mx https://orcid.org/0000-0002-7332-6146

Facultad de Humanidades; Universidad Autónoma de Baja California (México)

\section{Resumen}

La vida social familiar de clase media está organizada bajo patrones de socialización altamente tradicionales. Pocos son los espacios de uso de tiempo libre y ocio en la familia que abran posibilidades de construcción de vida social más armónicas y constructivas. El programa narrativo tradicional prescribe una desarticulación llamada "generacional" entre los hijos 
jóvenes y los padres de familia, en donde unos y otros se separan y pierden vínculo por tener menos o nulas actividades en común en la medida que los hijos se convierten en jóvenes. La vida social familiar, por tanto, va presentando elementos de erosión de su tejido social en el espacio doméstico, y poco a poco una desarticulación por las diferencia de actividades de tiempo libre y ocio, construidas socialmente para fortalecer este debilitamiento. La Ingeniería en Comunicación Social propone la convivencia pública y la música como tecnologías sociales eficaces para generar vida social que trae efectos enactivos que mejoren la calidad de las relaciones familiares, por lo que intervenir los espacios musicales públicos -fuera de los esquemas de segmentación jóvenes y adultos de clase media-, para recrearlos como acciones de construcción y fortalecimiento de vínculo jóvenes y padres en el ambiente social urbano.

Palabras clave: Familia; música; programas narrativos; Ingeniería en Comunicación Social; Tijuana.

Abstract

The middle class family social life is organized under highly traditional socialization patterns. There are few spaces for the use of free time and leisure in the family that open possibilities for the construction of more harmonious and constructive social life. The traditional narrative program prescribes a disarticulation called "generational" between young children and parents, where they separate and lose their connection by having fewer or no common activities as children become young. The social family life, therefore, is presenting elements of erosion of its social fabric in the domestic space, and little by little a disarticulation by the difference of activities of free time and leisure, built socially to strengthen this weakening. Engineering in Social Communication proposes public coexistence and music as effective social technologies to generate social life that has enactive effects that improve the quality of family relationships, so that public musical spaces intervene -apart from the segmentation schemes young and old. middle-class adults-, to recreate them as building actions and strengthening the bond between young people and parents in the urban social environment.

Keywords: Family; music; narrative programs; Social Communication Engineering; Tijuana.

Una de las características de mundo contemporáneo tiene que ver con las trayectorias históricosociales de la vida cotidiana que permanecen frente a otras que cambian. Lo nuevo y lo viejo

Question, Vol. 1, N. ${ }^{\circ}$ 63, julio-septiembre 2019. ISSN 1669-6581

Instituto de Investigaciones en Comunicación | Facultad de Periodismo y Comunicación Social | Universidad Nacional de La Plata 
conviven, esto ha generado un escenario particularmente cargado de incertidumbre y de contrastes. Las diversas dimensiones de la vida cotidiana enfrentan el desafío de encontrar los escenarios para ponernos de acuerdo para una mejor convivencia. El espacio social de relaciones entre los jóvenes y la familia se encuentra en esta situación, en donde predomina el debilitamiento de relaciones vinculares como familia, los grupos de pares, principalmente. Hacia fines del siglo XVIII y principios del XIX la familia (y casi en el mismo lugar la escuela) era el lugar social de preparación para la adultez. Pero en el siglo XX algo pasó, ese espacio social de formación social de orden doméstico comparte terreno con el espacio social del ocio y el tiempo libre, que poco a poco definirá prácticas y actividades propiamente juveniles. $Y$ la diferencia generacional abonó a esta desarticulación, lo hijos jóvenes y los padres tienen cada vez menos espacios de relación constructiva. El nuevo espacio social del tiempo de ocio y tiempo libre es marcado por su expresión de goce y evasión del mundo adulto, y el espacio doméstico no logra conectar con el espacio público.

Los espacios concretos de la vida social como la familia están en el centro de nuestra vida social del siglo XXI. Y ésta en el vórtice de la reproducción de la vida social desde la vida privada, con todo lo que ello implica en sus complicaciones, problemas y redefiniciones. Las formas de vida familiar tradicional son el lugar social desde dónde podemos comprender el sentido de la incertidumbre, y ver los espacios de posibilidad de construir otras formas de configuración social. La comunicación como constitución de lo social es terreno fundamental desde el cual se puede conocer y comprender a los sujetos y sus relaciones, pero también como dimensión para intervenir y mejorar las formas de relacionarnos. La vida familiar y los hijos jóvenes son objeto de interacciones complejas donde la familia lleva a cabo programas narrativos dominantes de convivencia entre padres e hijos jóvenes desde una visión adulta.

Pocos son los espacios sociales en donde los hijos jóvenes se articulan a un programa de vida familiar que no sea marcado por la diferencia generacional, que es más una construcción social que espacio de posibilidades. La familia forma sujetos sociales reproduciendo el esquema padres adultos e hijos jóvenes bajo figuras de dominación. La vida familiar sigue su curso en este esquema, y todas, o casi todas las prácticas socioculturales tienen esta característica de relación poco dialógica. La música y los gustos musicales (Bourdieu, 2010) son un patrón de vida sociocultural que se reproducen pero también marca claramente diferencias generacionales en el espacio social familiar.

Las manifestaciones y eventos musicales de tipo público son diseñados bajo este esquema generacional, delineados por la reproducción del viejo esquema de los gustos y las prácticas de los adultos que conducen la vida familiar frente a los gustos y prácticas de jóvenes que se van ajustando al Programa Narrativo Familiar. De ahí la necesidad de repensar y mirar desde la

Question, Vol. 1, N. ${ }^{\circ}$ 63, julio-septiembre 2019. ISSN 1669-6581

Instituto de Investigaciones en Comunicación | Facultad de Periodismo y Comunicación Social | Universidad Nacional de La Plata 
Gerardo Guillermo León Barrios. Vida familiar y música en el espacio social urbano. Programas narrativos e Ingeniería en Comunicación Social

Ingeniería en Comunicación Social otras formas de relación familiar donde la música sea un articulador, tanto en el gusto y apreciación en sus manifestaciones de tipo público.

Pasado y presente como sistemas de información y sistemas de comunicación. Familia, distancia generacional y programas narrativos

El conflicto generacional, como noción de la psicología clínica que aborda el asunto de un problema familiar como un problema creado por la diferencia de edad entre padres e hijos sólo desde la perspectiva intrapersonal, nos obliga a pensar que la distancia social que se crea por la diferencia de generación entre los miembros del grupo familiar es una construcción sociocultural y comunicacional, que obedece al diseño de un programa narrativo dominante apoyado por varios sistemas de información, como el de la familia tradicional, las industrias culturales, las prácticas culturales religiosas, las actividades y roles cotidianos, la escuela, entre otros. Si pensamos que los hijos adolescentes se encuentran en el proceso de su configuración subjetiva tan trascendente como la primera etapa de socialización (los primeros cinco años de vida), donde incorporan un universo simbólico complejo que modela su visión de la vida y el mundo, fundamentalmente dibujado en el espacio de vida familiar, nos mueve a pensar que la formación de individuos y sus competencias para su relación con sus padres y con sus pares puede intervenirse con otros referentes y experiencias que los haga mejores sujetos sociales para una vida en comunidad más constructiva.

La vida social del adolescente aparece como sorpresa y como problema en los sistemas familiares por la construcción social de los padres, con sus visiones y valoraciones de la vida adulta. El sistema de información padres de familia frente al sistema de información emergente de los hijos jóvenes tiene pocos elementos de colaboración constructiva. A los padres de hijos adolescentes se les hace difícil resolver el conflicto sin posibilidades, y no se generan relaciones a partir de una configuración dialógica que forme vínculos, la figura de relación entre sistemas de información de información es dominante.

Entendemos a los sistemas de información como el conjunto elementos que contienen información, y que configurados a manera de código, estructura, representan una visión específica del mundo que instaura reglas y formas de particulares de acción en el entorno, en donde hay otros sistemas de información, unos con mayor capacidad de dominar el entorno que otros, esto es, en su forma de hacer valer y hacer prevalecer esa particular visión del entorno. La familia es un sistema de información y por su capacidad prescriptiva, la familia es un sistema de información dominación que, regularmente, mantiene la hegemonía haciendo 
que predominen más los sistemas de reglas y de operaciones de la vida de sus individuos en el conjunto de hechos y acciones específicas.

El sujeto adolescente se encuentra en un ambiente sociocultural en una etapa de vida donde la familia no es necesariamente el eje de su cosmovisión, pero crea y recrea su mundo social con elementos de su espacio de formación como individuo. La vida familiar presenta, en buena medida, elementos de desarticulación en tanto el adolescente busca espacios de interacción menos autoritarias o jerárquicas (como lo es la familia), y va experimentando un tránsito de su espacio privado familiar al espacio público y amical, en donde éste le hace más sentido por su relación de par. El espacio social externo ha hecho que el adolescente se desarticule de la vida social familiar por la configuración jerárquica generacional que los padres ponen en práctica, lo que construye una relación de conflicto de poca asociación, interacción y sentido que va haciendo que va erosionando la convivencia familiar.

Al referirnos a los sistemas de comunicación, estamos hablando de procesos de sistemas de información de carácter dialógico y de mutua afectación. Los sistemas son capaces de entrar en contacto con otros sistemas de información, por los que la capacidad dominante de algunos se ve afectada por interacciones y formas de colaboración entre los individuos y sus sistemas de información. Las prescripciones que establecen las reglas y formas de orden de los sistemas de información son alteradas, transformadas, evolucionadas ante la conciencia de necesidad de cambio. Los sistemas de información, por tanto, pueden pasar a un nivel de configuración en donde la valoración del mundo se afecta para favorecer la comunicación. Así, podemos decir que existe un sistema de comunicación. La familia, al reproducir socioculturalmente a la sociedad, ejecuta elementos de un programa narrativo dominante, parte de lo que debe ser la familia como modelo social, y sus procesos de socialización son puestos en prácticas con elementos tradicionales, como la jerarquía, la autoridad y los roles que tienen como fundamento la diferencia generacional. Los hijos pertenecen a gustos y prácticas que no son de la generación de los padres, donde los gustos y las prácticas fueron diferentes. El pasado como sistema de información dominación frente al presente, un sistema de información con posibilidades de emergencia de sistemas de comunicación. La ejecución del programa narrativo dominante está mediado por el programa narrativo pragmático, la vida social familiar se realiza bajo circunstancia de contingencia, con poca creatividad que permite pocas o nulas actividades de vida familiar donde el sistema de información de los hijos esté en el centro, o en la figura de colaboración con el sistema de información padres. De ahí que todas las actividades que se realizan en la familia son establecidas por los padres y los hijos construyen sus propios espacios. Las actividades en común son una mediación del programa narrativo dominante. En este patrón se va definiendo y construyendo el gustos musical y las actividades 
asociadas a ello. Los hijos definen sus gustos musicales y sus prácticas en el espacio extra familiar, a partir de sus sistemas de información, no el de los padres. La música, como gusto, así como sus prácticas aparecen como un sistema de información dominación que instaura gusto y tipos de prácticas. El sistema de información musical de los padres es un factor de configuración de conflicto y distancia social, la inminente separación generacional de gustos y prácticas musicales de los padres y de gustos y prácticas musicales de los hijos.

La música en familias de clase media como experiencia común. De los programas narrativos hacia el desarrollo de la cibercultura

Esta propuesta de Ingeniería en Comunicación Social de la música se encuentra asociado al tema de la familia estudiando un caso en el suburbio en Playas de Tijuana, Tijuana, Baja California, entre 2013 y 2014. La configuración comunicológica de la familia parte del principio de que todo grupo social familiar ha desarrollado formas de vida familiar en ciertas trayectorias y con posibles tendencias, entendiendo a la comunicación como la dimensión que hace posible lo social.

El primer resultado está construido a partir de las unidades básicas de investigaciónobservación con los elementos de las dimensiones comunicológicas y las configuraciones sistémicas de las formas de vida familiar, que es lo que hace un Programa Narrativo Familiar, esto es un análisis de cómo en un tipo de familia y sus actores ejecutaron acciones con objetos sociales y de comunicación definidos por el fin sistémico familiar o la función de vida familiar para sostenimiento del sistema social, según las categorías de difusión, expresión, interacción y estructuración. La descripción hace visibles en qué dimensión se desarrollaron actividades, situaciones y prácticas de vida familiar, y si es alguno o algunos tipos de sistemas de información o si es alguno o algunos tipos de sistemas de comunicación. El esquema analítico de las dimensiones es la base para el segundo nivel de análisis, el comunicológicocibercultural.

En el análisis comunicológico-cibercultural se representan sistémicamente la vida social familiar en un sentido que ya podemos nombrar comunicológico. De ello se ha sintetizado un paquete de cinco modelos básicos en el caso familias de Playas de Tijuana, o Modelos comunicológicos familiares. El modelo 1, que es una Familia con un Sistema de Información de Dominación (FSID); el modelo 2, modelo Comunicológico de Familia con un Sistema de Información de colaboración (F-SIC); modelo 3, familia con un Sistema de Información de dominación y un Sistema de Comunicación emergente (F-SIDySC); modelo 4, constituido por una familia con un 
Sistema de Información de Colaboración y un Sistema de Comunicación emergente ( $F$ SICySC); finalmente, el modelo 5 que sintetiza a una familia con un Sistema de Información de Colaboración y con un Sistema de Comunicación de Colaboración (F-SICySCC).

En el estudio de caso de familias de clase media del suburbio Playas de Tijuana, los modelos más comunes son el 1 (F-SIC) y el 2 (F-SIC), el 3 (F-SIDySC) en menor medida. El modelo 4 (F-SICySC) y 5 (F-SICySCC) son apenas visibles, lo cual nos dice que desde el punto de vista comunicológico los Sistema de Información Dominación prevalecen frente a Sistemas de Información de colaboración que generen Sistemas de Información Comunicación con rasgos de colaboración, pero aparecen.

Con este diagnóstico comunicológico observamos desde la configuración cibercultural características de comunicación más favorecidas o menos desarrollas en la vida social familiar, el origen de muchos conflictos que no favorecen el desarrollo de una familia con niveles de bienestar importantes desde sus interacciones y comportamientos, que se manifiestan en acciones de tipo contacto, interacción, conexión, vínculo o enacción en diferentes niveles y combinaciones. Esto no permite explicar cómo una forma de vida familiar (Programa Narrativo Pragmático Familiar) se ha desarrollado con prácticas o dinámicas de comunicación que construyen o no vida social constructiva y colaborativa, según las formas de relación que más practican o han practicado sus actores sociales. En el caso del diagnóstico en Playas de Tijuana, tenemos tres modelos de configuración cibercultural siguientes:

Tipo 1. Familia con una configuración cibercultural de interacción (FCC-I). Vida familiar con configuración de comunicación y sus operaciones ciberculturales más desarrolladas en el nivel de la interacción.

Tipo 2. Familia con una configuración cibercultural de interacción y elementos de conexión (FCC-IC). Tipo de familia donde el Programa Narrativo Pragmático ha tenido mayor trabajo de convenios y acuerdos para favorecer un estado de relación familiar con más empatía y conciliación.

Tipo 3. Familia con una configuración cibercultural de interacción con elementos de conexión y vínculo (FCC-ICV). Vida familiar que tiene elementos de vínculo, y que en su configuración cibercultural ha llegado a un grado de compromiso y responsabilidad alto.

En el programa narrativo familiar de este caso, los elementos de conexión y vínculo son menos favorecidos, que es donde se genera y refuerza el conflicto generacional. La erosión en la 
relación padres e hijos dados que los sistemas de información entran escasamente en colaboración. De ahí el llamado conflicto generacional en las formas de vida familiar. La música, en su práctica de escuchar y compartir, es un sistema de información está en el centro de la desarticulación. El sistema de información música emerge en el sistema familiar desde el marco de la separación que crea la diferencia generacional. La conexión y el vínculo a través y con la música no son elementos de la configuración comunicológica de la familia. Si se sigue el Programa Narrativo Dominante, que son los casos de la mayor parte de las familias del caso Playas de Tijuana, la práctica de formar un gusto y de escuchar como música es parte del sistema de información dominación, esto es, lo padres incorporan a la música desde sus parámetros y experiencias, desde la trayectoria del pasado y la visión adulta, desde la perspectiva de una generación. Por otro lado, el hijo adolescente va formando gusto y relación con la música fuera de sistema familia, sino con su mundo externo. Sus elementos de conexión y vínculo dentro de la familia no toman sentido, y si lo tienen en el espacio extra familiar. La construcción de un sistema de comunicación con acciones y emociones positivas es escasa, o existe en muy pocos niveles de interacción. Y aun así a veces no se encuentran. Por ello creemos pertinente aprender a construirlas bajo la base de sistemas de comunicación de colaboración donde la emocionalidad es base de la construcción de vida social familiar con mejores niveles de bienestar subjetivo en prácticas de compartir y degustar música como comunimétodo que genera condiciones para construir bienestar familiar y contribuir al bienestar general, en colaboración con los demás sistemas de información y comunicación.

\section{Convivencia privada y pública en la construcción de vida familiar vincular}

La construcción de sistemas de comunicación familiar con niveles altos de vínculo tiene como base el conocimiento científico de la comunicología e Ingeniería en Comunicación Social de la música en su práctica de escuchar y compartir colectivamente, desde donde se propone intervenir las formas de vida familiar en sus tres tipos de operación (instaurar elementos de cambio, acompañar y dar sustento a los ya en desarrollo y/o ajustar elementos sistémicos para pasar de un estado a otro), como una tecnología social tiene un gran poder para suscitar emociones de colaboración y vida social constructiva que educan para la ciudadanía (Bisquerra, 2011). Los espacios sociales familiares de sistemas de información que se han separado o han no han tenido contacto o relación con el fin de generar bienestar personal y social se pueden redireccionar en sus tendencias hacia trayectorias posibles. La vida social familiar no puede negar que el sistema de información padres se desarrolla bajo un poder 
sobre el sistema de información hijos, por lo que el desarrollo de estas competencias de colaboración a través de situaciones en donde se comparte y escucha música, ya sea en espacios públicos o en el ámbito doméstico, produce efectos emocionales y vinculares en la familia con efectos en la distancia generacional que desarticula el sistema familiar, el llamado "espacio familiar" es un terreno social con altas posibilidades de ser intervenido desde la Ingeniería en Comunicación favoreciendo escenarios posibles de cambios, comportamientos y formas de interacción social en diferentes estadios y trayectorias de lo social comunicológico en la familia

En el diagnóstico ha identificado tendencias y trayectorias y propone diseñar estrategias de posibles acciones que implican tecnologías sociales y comunimétodos (Galindo Cáceres, 2011). La tecnología social la consideramos como el elemento que media entre el diseño del concepto ingenieril y la actividad que llevará a realización del diseño en el sentido práctico. Desde el punto de vista de la Ingeniería en Comunicación Social, vemos a la combinación fiesta, convivencia y música como tecnologías sociales eficaces para generar vida social que trae efectos enactivos que mejoran la calidad de las relaciones familiares. $Y$ vemos a los comunimétodos como la matriz etnometodológica de la vida social familiar en la vida cotidiana como las microacciones que suscitan los espacios y momentos de escuchar, compartir e interactuar a través de la música en espacios públicos y privados. La vida social familiar tiene espacios limitados de vida social, la participación de grupo en el ambiente social público está centrada bajo sistemas de información consumo y religión en sociedad mexicana contemporánea. De acuerdo a la hipótesis de desarrollo de esta propuesta, la activación de la convivencia de padres a hijos adolescentes en actividades musicales públicas crea formas de relación conexión y vínculo, en tanto se comparten sentidos y significados superando la construcción social de actividades y eventos de tipo público que segmentan por generaciones. El sistema familiar tiene capacidad de adaptarse a los cambios y situaciones. La familia funciona con niveles de sustentabilidad, esto es, sin conflicto, cuando se crean espacios de conexión y vínculo donde sus actores entran en un nivel compromiso de convivencia y colaboración como miembros del sistema, recreando y subvirtiendo los patrones de comportamiento prescritos por el Programa Narrativo Dominante y la relación entre sistemas de información colaborativos. En este sentido, la participación y convivencia los miembros de un grupo familiar en eventos de música de sin la mediación de la diferencia generacional en el gusto y sus prácticas, puede llevar a la configuración de vida familiar en el tipo comuniconómico 4 y 5 (León, 2015). En el Tipo comuniconómico de Familia Alterna Emergente, se sintetiza en un desarrollo de vida familiar emergente, el cual sigue algunos patrones del Programa Narrativo Dominante, pero cambia y se reconfigura en el desarrollo del 
Gerardo Guillermo León Barrios. Vida familiar y música en el espacio social urbano. Programas narrativos e Ingeniería en Comunicación Social

Programa Narrativo Pragmático según transformaciones de la vida social, comunicológicamente constituida por un Sistema de Información de Colaboración y un Sistema de Comunicación Emergente, que tiene elementos de colaboración con otros Sistemas de Información, y su configuración cibercultural es de Interacción con elementos de Conexión y de Vínculo. En este caso, la música, tanto el gusto como sus prácticas, son un elemento articulador que no depende del Programa Narrativo Dominante, los padres escuchan, pero sobre todo, participan de actividades y eventos de música con espacios alternativos de convivencia (Haley, 2008) a los que prescribe el modelo tradicional. Las actividades compartidas construyen interacciones de conexión y vínculos en donde padres e hijos se asociación por algo que da sentido en grupo, en común.

El nivel más desarrollado, Tipo comuniconómico de Familia Alterno Posible, que está sintetizado en una vida familiar que se realiza cercano al Programa Narrativo Dominante, que se construye en los cambios y transformaciones de la vida social que favorecen al Programa Narrativo Pragmático, comunicológicamente constituida por un Sistema de Información de Colaboración y con un Sistema de Comunicación de Colaboración, hay colaboración con otros Sistemas de Información, su configuración cibercultural es de Interacción con elementos de Conexión y de Vínculo. Este modelo es el más deseable en cuanto a la construcción de vida familiar con niveles altos de conflicto generacional, en tanto que la relación de los sistemas de información son de colaboración intra y extra familiar, no se depende de la prescripción del Programa Narrativo Dominante, sino que la recreación pragmática es alta y fundamenta. En este caso, la convivencia a través del gusto y las prácticas musicales son un dinamizador de las configuraciones de conexión y vínculo. El acuerdo de comunidad familiar es claro y vigente, se comparte el sentido de la participación en eventos y espacios de convivencia con la música de manera consensada.

Música y sistema de comunicación colaboración familiar. Ocio, oferta musical y gentrificación

Los estudios y aplicaciones de la noción de gentrificación aluden a otras formas de habitar las ciudades. La discusión centrada en la habitabilidad de los espacios de los centros de las ciudades y grandes urbes recientemente contempla otros elementos relacionados con las reapropiaciones de espacios.

Otras lecturas y análisis de la gentrificación están incorporando el valor de estos espacios a partir de la oferta cultural que detonan la construcción de los procesos de gentrificación. Este

Question, Vol. 1, N. ${ }^{\circ}$ 63, julio-septiembre 2019. ISSN 1669-6581

Instituto de Investigaciones en Comunicación | Facultad de Periodismo y Comunicación Social | Universidad Nacional de La Plata La Plata | Buenos Aires | Argentina

Página 10 de 14 
proceso está integrando en sus aplicaciones urbanísticas los asuntos de usos del tiempo libre y el ocio de los actores ahí habitan y los que asisten al nuevo escenario urbano. La oferta cultural como activador de dinámicas y procesos de renovación en el espacio público tienen posibilidades de abrir no sólo opciones de económico, sino también de reconfiguración de la vida social urbana, y donde las prácticas de vida familiar se ajustan bien a nuevo modelo de vida pública.

El nuevo espacio social urbano y su nuevo "capital cultural" es un articulador del nuevo espacio urbano como sistema de información con posibilidades constructivas para configurar sistemas de comunicación. La vida social contemporánea y sus formas de vida social familiar con Programas Narrativos Pragmáticos requieren de activadores emocionales de orden colectivo y público en los procesos de gentrificación como son actividades y eventos musicales sin segmentaciones generacionales. La vida social pública segrega por categorías y niveles de consumo. La construcción de un sistema de comunicación del espacio público en el que se comparte la vida pública y acceso y disfrute de música incentiva procesos de articulación social que desactivan la individualidad y las distancias generacionales. La familia debe puede reajustar su Programa Narrativo Dominante (cultura tradicional) e incorporar al espacio urbano compartido situaciones que posibiliten sistemas de comunicación de vida social colectiva en espacios o procesos de gentrificación.

Las expresiones artísticas, como la música compartida y disfrutada en público genera efectos de renovación y reforzamiento de tejido social y de otras formas de ciudadanías. El espacio gentrificable recupera la noción de sujetos colectivos, sin embargo es poco lo que se reconoce a los sistemas de vida familiar que potencia la recuperación y resignificación de la nueva cultura urbana, por un lado, y por el otro, la se reconoce poco la importancia de espacios públicos con oferta cultural musical como tecnología social de alto valor social por sus efectos de construcción de vida social colectiva y ciudadana. El lugar de cultura, y en particular de eventos musicales, son activadores de la gentrificación, pero también son nuevas formas de configuración de comunicación social. La incorporación del sistema familia como otro actor de este proceso da posibilidades de vinculación en dos sentidos (Smith, 2012), introyecta reajustes a su Programa Narrativo Pragmático en relaciones padres e hijos debilitando la distancia generacional por gustos y preferencia musicales, y proyecta alternativas de construcción de sujetos colectivos por la experiencia de compartir y disfrutar música entre familia, entro otros participantes y con los creadores musicales; todo ello como un gran sistema de comunicación que transforma el tejido social urbanos.

La familia es un sistema sociocultural en un continuo proceso de equilibrio y cambio, un equilibrio entre lo tradicional y lo moderno, y cambio entre lo heredado por la tradición y lo 
emergente de la cultura contemporánea. Así también sucede con los sistemas de información y sistemas de comunicación como ambientes socioculturales en el que se desenvuelven. Las posibilidades de cambio en actividades asociadas a la música y eventos de interacción públicos son una posibilidad de modificar y trascender los sistemas jerárquicos, las diferencias generacionales, las distancias individuales de consumo así como roles y reglas con el propósito de redefinirla convivencia como grupo complejo y sus vínculos familiares.

\section{Bibliografía}

Abalos, C. (2013). El taller, un dispositivo para el encuentro y la reflexión. Buenos Aires: La Crujía.

Agier, M. (2011). Antropologia da cidade. Sau Paulo, Brasil: Terceiro Nome.

Alberto, R. (2005). Estrategias de comunicación. Barcelona: Ariel.

Alvarado, I.; Cuenca, A. y Gómez, T. (13/07/2010). La nueva Tijuana. El Universal, p-22.

Barajas, M. y Méndez Mungaray, E. (1993). Consideraciones generales sobre población, desarrollo y medio ambiente, el caso de Tijuana, Baja California. Estudios Fronterizos, 29, UABC, México.

Bauman, Z. (2003). Comunidad. En busca de seguridad en un mundo hostil. Madrid: Siglo XXI.

Bauman, Z. (2003). Modernidad líquida. México: Fondo de Cultura Económica.

Beck-Gernsheim, E. (2003). La reinvención de la familia. En busca de nuevas formas de convivencia. Barcelona: Paidós.

Bertalanffy, L. von (1976). Teoría general de los sistemas. México: Fondo de Cultura Económica.

Bisquerra, R. (2011). Educación emocional. Propuestas para educadores y familias. España: Desclée de Brower.

Bourdieu, P. (2010). El sentido social del gusto. Elementos para una sociología de la cultura. Argentina: Siglo XXI.

Buxarráis, M. y Zeledón, M. (2007). Las familias y la educación en valores. Retos y perspectivas actuales. Barcelona: Editorial Claret.

Cicalese, C. (2010). Comunicación comunitaria. Apuntes para abordar las dimensiones de la construcción colectiva. Buenos Aires: La Crujía.

De la Calle, L. y Rubio, L. (2010). Clasemediero. Pobre no más, desarrollado aún no. México: Centro de Investigación para el desarrollo. 
Del Fresno, M. (2011). Retos para la intervención social con familias en el siglo XXI. Consumo, ocio, cultura, tecnología e hijos. Madrid: Editorial Trotta, Universidad Nacional de Educación a Distancia.

Estrada, L. (2010). El ciclo vital de la familia. México: De Bolsillo.

Galindo Cáceres, L. J. (2006). Cibercultura. Un mundo emergente y una nueva mirada, CNCAInstituto mexiquense de la cultura, Toluca.

Galindo Cáceres, L. J. (2007). Comunicología y Epistemología. El tiempo y las dimensiones sistémicas de la información y la comunicación. En Estudios sobre las Culturas Contemporáneas, Época II, Vol. XIII, Número 26, Diciembre 2007, Universidad de Colima, Colima.

Galindo Cáceres, L. J. (coordinador). (2008). Comunicación, Ciencia e Historia. Fuentes científicas históricas hacia una Comunicología Posible. Madrid: McGraw Hill.

Galindo Cáceres, L. J. (2011). Ingeniería en Comunicación Social y Promoción Cultural. Sobre Cultura, Cibercultura y Redes Sociales. Argentina: Homo Sapiens, Universidad Nacional del Rosario, Benemérita Universidad Autónoma de Puebla.

Galindo Cáceres, L. J. (2012). Ingeniería en Comunicación Social y Deporte. México: Instituto de Altos Estudios en Deporte, Cultura y Sociedad.

Galindo Cáceres, L. J. (2012). Exploración de la historia de los conceptos de clase social y estrato social. En López Romo, H. (2012). Ilustración de los Niveles Socioeconómicos en México. México: Instituto de Investigaciones Sociales.

Galindo Cáceres, L. J. (2014). Ingeniería en Comunicación Social. Hacia un Programa General, Puebla: Instituto de Ciencias de Gobierno y Desarrollo Estratégico-Benemérita Universidad Autónoma de Puebla.

Galindo Cáceres, L. J. (coordinador). (2015). Ingeniería en Comunicación Social y Familia. Cuadernos Artesanos de Comunicación, 79, Latina, La Laguna, Tenerife.

Galindo Cáceres, L. J. y Islas Carmona, O. (coordinadores) (2015). Ingeniería en Comunicación Social y Comunicación Estratégica. Cuadernos Artesanos de Comunicación, 75, Latina, La Laguna, Tenerife.

Giddens, A. (2000). Un mundo desbocado. Los efectos de la globalización en nuestras vidas. Madrid: Taurus.

Haley, J. (2008). Terapia para resolver problemas. Nuevas estrategias para una terapia familiar eficaz. Buenos Aires: Amorrortu.

López Romo, H. (2012). Ilustración de las familias en México. Con base en la tipología desarrollada por El Instituto de Investigaciones Sociales. México: Instituto de Investigaciones Sociales.

Question, Vol. 1, N. 63 , julio-septiembre 2019. ISSN 1669-6581

Instituto de Investigaciones en Comunicación | Facultad de Periodismo y Comunicación Social | Universidad Nacional de La Plata 
Luhmann, N. (1998). Sistemas sociales: Lineamientos para una teoría general. Barcelona: Universidad Iberoamericana Santa Fe, Antrhopos y Pontificia Universidad Javeriana.

Macías, N. y Cardona, D. (2007). Comunicometodología. México: UIC.

Marc, E. y Picard, D. (1992). La interacción social. Cultura, instituciones y comunicación. España: Paidós.

Martín Serrano, M. (1994). La producción social de comunicación. España: Alianza Universidad.

Massoni, S. (2007). Estrategias. Los desafíos de la comunicación en un mundo fluido. Rosario: Homo Sapiens Ediciones.

Morán, M. L. y Benedicto, J. (2000). Jóvenes y ciudadanos. Madrid: Instituto de la juventud.

Smith, N. (2012). La nueva frontera urbana. Ciudad revanchista y gentrificación. España: Traficantes de Sueños.

Sunkel, G. (2006). El papel de la familia en la protección social en América Latina. Chile: CEPAL.

Tuirán, R. (1999). Estructura familiar trayectorias de vida en México. En Gómez, C. (Comp.). Procesos sociales, población y familia. México: Facultad Latinoamericana de Ciencias Sociales (FLACSO).

Valenzuela, J. M. y Salles, V. (1998). Vida familiar y cultura contemporánea. México: Conaculta.

Wortman, A. (Coord). (2003). Pensar las clases medias. Buenos Aires: La Crujía. 